\title{
Management of Candida infections in liver transplant recipients: current perspectives
}

\author{
This article was published in the following Dove Press journal: \\ Transplant Research and Risk Management \\ 7 July 2014 \\ Number of times this article has been viewed
}

Pushpalatha B

Lingegowda ${ }^{1-3}$

Ban Hock Tan 1,2,4,5

'Department of Infectious Diseases, Singapore General Hospital, ${ }^{2}$ DUKENUS Graduate Medical School, ${ }^{3}$ Yong Loo Lin School of Medicine, National University of Singapore, ${ }^{4}$ SingHealth Internal Medicine Residency Program, ${ }^{5}$ Faculty of Medicine, National University of Singapore, Singapore

Correspondence: Pushpalatha B Lingegowda

Department of Infectious Diseases, Singapore General Hospital,

Academia Level 3, 20 College

Road, Singapore 169856

Tel +65 632I 3479

Fax +65 62275247

Email pushpalatha.bangalore.lingegowda@ sgh.com.sg

\begin{abstract}
Liver transplantation has emerged as a widely accepted lifesaving therapeutic option for many patients with a variety of liver diseases. Improved surgical and medical management has led to significant improvements in post-transplant survival rates with a 1 year and 5 year patient survival of $87 \%$ and $73 \%$, respectively. A high mortality rate due to infections during the first post-transplant year persists. Invasive candidiasis is recognized as a significant problem associated with high morbidity and mortality. Recent surveillance data has helped to understand the changes in the epidemiology and the evolving trends in the use of antifungal agents for prophylaxis and treatment combined with the challenges of managing these invasive fungal infections, which has led the transplant community to explore the best management strategies. The emergence of resistant fungi and excess costs in managing these invasive fungal infections has added to the complexities of management. In this context, current perspectives in the management of Candida infections in liver transplant recipients will be reviewed.
\end{abstract}

Keywords: Candida infections, management, liver transplant

\section{Introduction}

The field of liver transplant has progressed remarkably since the first attempt at orthotopic human transplant in 1963 by Thomas Starzl in the US. Better understanding and improved outcomes have led to wider indications for liver transplant leading to an increased group of immunocompromised hosts who are now living longer and are at increased risk of infective and noninfective complications. Infections have been recognized as a frequent complication with bacterial pathogens playing a major role in the immediate postoperative period followed by viral and fungal organisms. ${ }^{1-5}$ Among fungal infections, Candida spp. and Aspergillus spp. are the most common pathogens and are described as the most devastating complications contributing to significant morbidity and mortality. ${ }^{6}$ Recognizing the factors which lead to these infections and developing appropriate and timely strategies to prevent, diagnose, and manage these invasive fungal infections (IFIs) will be an ongoing challenge faced by transplant teams. A survey on antifungal management practices has revealed the difficulties in making uniform recommendations as there are variations in practice at different transplant centers. ${ }^{7}$ Local epidemiological data should guide decisions on choice of antifungal agents and help formulate local guidelines. ${ }^{8}$ The epidemiological changes, recognized risk factors, clinical features, and management approaches currently available in managing Candida infections in liver transplant recipients will be discussed. 


\section{Epidemiology}

Surveillance reports suggest changes in the epidemiology of Candida infections among liver transplant recipients. The overall incidence of Candida infections has decreased compared to the early transplant years, but Candida spp. continue to be the most common pathogen. ${ }^{9}$ However, even with evolution of transplant practices, IFIs carry a high mortality rate. ${ }^{10}$ Increasing incidence of infections due to non-albicans Candida spp. and a shift toward late occurrences, well beyond the traditional risk period, are being reported.

The incidence of Candida infections has decreased from $30 \%-50 \%$ in the early years of transplant history to approximately $10 \%$ in the early 21 st century, to the current estimates of $<5 \% .{ }^{11,12}$ The majority of earlier reports are from single-center studies with relatively small sample sizes, although recently multicenter collaborations have given us a better overview.

In an early six-center study of invasive candidiasis (IC) in liver transplant recipients, Husain et al compared 35 patients with IC with 69 patients without IC. ${ }^{13}$ No denominator data are provided so the incidence could not be calculated. Candidemia accounted for $40 \%$ of IC, and peritonitis another $40 \%$. The median time to infection was 13.5 days (range 1-4,109 days); 78\% of infections occurred in the first 3 months after transplant. In this series, the species identified were C. albicans (65\%), Candida glabrata (21\%), Candida tropicalis (9\%), Candida parapsilosis (3\%), and Candida guilliermondii (3\%).

More recently, larger multicenter studies on IFIs in solid organ transplant patients have revealed subtle changes in Candida epidemiology. The Transplant-Associated Infection Surveillance Network surveyed multiple US centers to understand IFIs in solid organ transplant recipients. ${ }^{12}$ The Prospective Antifungal Therapy Alliance (PATH Alliance) is another study that aimed to collect data on IFIs in the US; a subset of the study, focusing on solid organ transplant recipients, was published by Neofytos et al. ${ }^{14}$

In the PATH Alliance report, IC was the most frequently identified IFI overall, accounting for 59\% of IFIs. Among the liver transplant recipients, Candida accounted for $78.7 \%$ of IFIs, with C. albicans contributing to $45 \%$ of all the Candida spp. identified. ${ }^{14}$ In terms of median day of diagnosis, IC tended to be an early complication, generally diagnosed within the first 6 months of transplant. Among liver transplant recipients, IC was diagnosed at a median of 155 days after transplant, although the range was large ( $0-5,626$ days). This contrasts with invasive aspergillosis, which was generally a later complication. ${ }^{14}$

According to Transplant-Associated Infection Surveillance Network data, Candida was also the most common IFI in organ transplant recipients - with a 12-month cumulative incidence of $1.9 \%$, it far surpassed other fungal pathogens as a cause of IFI. ${ }^{12}$ Candidemia was identified as the most common type of IC in this series. Among liver transplant recipients, candidiasis accounted for $68 \%$ of IFIs over the 3 -year surveillance period. In this study, the median time to diagnosis of IC was 103 days.

It is noteworthy that in the more recent studies (Transplant-Associated Infection Surveillance Network and PATH Alliance) the median times to the diagnosis of IC were greater than that in the earlier study of Husain et al. ${ }^{13}$ Although the accuracy of larger numbers might be contributory, improvements in surgical technique have probably played an important role in reducing infective complications. ${ }^{8}$ The role played by antifungal prophylaxis (particularly newer agents such as the echinocandins) in delaying the time to IC has not been fully investigated. Singh et al have shown that more liver transplant centers are practicing prophylaxis, with caspofungin being one of the more popular agents. ${ }^{7}$ The duration of prophylaxis varied, but few centers used them for $>3$ months.

\section{Risk factors and the timing of Candida infections}

Liver transplant recipients have multiple risk factors for Candida infections and the vulnerability and incidence are influenced by host, environment, and fungal factors. Host factors include immunosuppressants (eg, dose and duration), breaches in the anatomical barriers (eg, from the surgical procedure), environmental exposures (health care related or community acquired), and fungal factors (eg, resistance to prophylaxis used). ${ }^{15}$ Main risk factors of liver transplant recipients for IC identified are retransplantation, creatinine level $\geq 2.0 \mathrm{mg} / \mathrm{dL}$, choledochojejunostomy, intraoperative use of 40 units of blood products, prolonged intraoperative time ( $>11$ hours), and fungal colonization.

Despite these many risk factors, it is thought that other factors have probably contributed to lower rates of IC documented in modern-day practice. These include refinements in surgical technique, improved understanding of immunosuppression, improved cytomegalovirus prevention strategies, and antifungal prophylaxis. However, routine antifungal prophylaxis without risk stratification has been implicated for the increasing trends in non-albicans Candida spp. and resistant Candida spp. . $^{13,16,17}$

Candida spp. frequently colonize several anatomical sites, which include the skin, oral cavity, gastrointestinal tract, and the vagina, and constitute the most common fungal pathogens 
to affect humans. Nosocomial infections due to Candida spp. have increased in vulnerable patients, critically ill patients in the medical and surgical intensive care units, and solid organ transplant and hematopoietic stem cell transplant recipients. There is a global increase in the frequency of candidemia and IC. ${ }^{18}$ Crude mortality rates of Candida bloodstream infection in intensive care unit patients were higher $(42.6 \%)$ compared to the more prevalent Gram-positive (25.3\%) and Gram-negative bloodstream infection (29.1\%). ${ }^{19}$

Altered host immunity is recognized as the principal factor for the opportunistic pathogen; however, adherence of the microbe to host cell and biofilm formation, secretion of hydrolytic enzymes which leads to tissue invasion, ability to switch from yeast and filamentous forms which leads to invasion, and switching phenotypes are a few of the many virulence factors which are being explored in Candida spp. ${ }^{20-22}$

Among liver transplant recipients, C. albicans is the most common isolate followed by other species of Candida. An increase in the proportion of non-albicans Candida spp. has been noted over the last 2 decades. In studies published from 1995-2003, C. albicans accounted for 55\% of proven infections; in studies published from 2004-2007, C. albicans accounted for $45 \%$ of proven and probable IFIs. Among the non-albicans Candida spp, C. glabrata was the dominant species followed by Candida krusei, C. parapsilosis, and C. tropicalis. ${ }^{14,17}$ C. glabrata and C. krusei were commonly isolated from patients who had received antifungal therapy. ${ }^{14}$ Fluconazole resistance in $C$. albicans, C. tropicalis, and C. parapsilosis was low $(<1 \%){ }^{23}$

The temporal association of infections occurring in organ transplant recipients following the initiation and continuation of immunosuppressants is well described, and the clinical timetable for infections has helped clinicians in generating differential diagnosis while evaluating infections in posttransplant patients. It has also been used as a tool to formulate preventive strategies. ${ }^{24}$ Evolving trends suggest a change in the timing of these infections; recent data show IC are occurring later than the traditional timeline of 1 month for nosocomial fungal infection. ${ }^{25}$ The impact of cytomegalovirus and fungal prophylaxis contributing to the shift in the timelines of these fungal infections has been suggested. ${ }^{26}$

\section{Clinical aspects of Candida infections}

Mucocutaneous candidiasis and IC represent different ends of the spectrum of infections caused by Candida. ${ }^{27}$ Mucocutaneous candidiasis involves the skin and its appendages and the mucous membranes of the oropharynx, esophagus, non-esophageal gastrointestinal tract, respiratory tract, and genitourinary tract. IC includes candidemia (Candida spp. isolated from blood cultures) and organ involvement (tissue sites infection).

Abdominal organ transplant recipients in general, and liver transplant recipients in particular, exhibit unique susceptibility to Candida infections. ${ }^{15}$ In the PATH Alliance study, which evaluated 429 adult solid organ transplants with IFIs, IC manifested as candidemia, abdominal infection, lung infection, and skin involvement. ${ }^{14}$ Among liver transplant recipients, postoperative infections such as intraabdominal (peritonitis, biliary tract, abdominal abscess, enteritis), skin and soft tissue infections, and infected bilomas are complications associated with Candida infections which are unique, difficult to manage, and associated with significant morbidity and mortality. ${ }^{28-31}$

Recognizing infections in transplant recipients is difficult; clinical signs and symptoms are often not specific and might be muted. ${ }^{3,11}$ One-third of patients with infected hepatic fluid collections (bilomas) were asymptomatic. ${ }^{28}$ Fever is a nonspecific symptom. At the same time, differentiating infective from noninfective causes requires thorough clinical evaluation, diagnostic procedures, and radiologic imaging to arrive at an appropriate diagnosis. For example, allograft rejection, drug fevers, postoperative hematoma, and tissue infarcts can mimic infections and lead to diagnostic dilemmas. ${ }^{32}$

\section{Diagnosis of Candida infections}

Candida spp. are known colonizers of humans - interpreting a positive culture from a nonsterile site is notoriously difficult.

Guidelines do exist to help categorize patients with IFIs with varying levels of probability. The revised definitions of invasive fungal disease from the European Organization for Research and Treatment of Cancer/IFI Cooperative Group and the National Institute of Allergy and Infectious Diseases Mycoses Study Group Consensus Group were formulated primarily to "facilitate the identification of reasonably homogeneous groups of patients" for research purposes. ${ }^{33}$ In an earlier edition of the guidelines, the authors stress that the guidelines "should not be taken as strict rules for making or excluding the diagnosis of an IFI in clinical settings". ${ }^{34}$

Diagnosis of candidemia and IC has traditionally been based on clinical suspicion, suggestive signs and symptoms, and cultures of blood, imaging, and biopsy of the suspected lesion for cultures and histopathology. They remain as important as ever, but nonculture techniques in the form of biomarkers may improve our ability to diagnose candidemia 
and IC. The latest European guidelines on diagnostic procedures of choice for the diagnosis of candidemia put $1 \rightarrow 3$ - $\beta$-D-glucan (BDG) and the mannan/antimannan assays in the "recommended" category. ${ }^{35}$ Data for their use in liver transplant recipients, however, remain scarce.

Blood cultures are crucial for the diagnosis of candidemia. Although blood cultures are said to be insensitive with an approximately $50 \%$ yield, their yield may be optimized with adherence to consensus recommendations, eg, by sending adequate volumes of blood and doing multiple sets. ${ }^{35}$ In addition, the time taken for species identification following positive blood cultures can be quite prolonged, leading to a delay in effective antifungal therapy. Cultures, however, are relatively inexpensive and allow for drug sensitivity testing. Peptide nucleic acid fluorescence in situ hybridization and matrix-assisted laser desorption ionization-time of flight mass spectroscopy are techniques developed to decrease the time to species identification and have been successfully applied to Candida as well as bacteria. ${ }^{36,37}$

BDG is a component of the cell wall of many types of fungi. Measurement of BDG in serum has been associated with candidemia and aspergillosis. BDG, however, is not released by Cryptococcus or Mucormycosis. BDG measurement may be considered an aid in the diagnosis of fungemia; in particular, candidemia and IC. In a series focusing only on patients in the intensive care unit, Posteraro et al drew blood for cultures and BDG at the onset of sepsis. In their series, 13 of 14 patients with candidemia or IC were positive for BDG. There were few false positives; hence, the test in the hands of these investigators had sensitivity and specificity in excess of $93 \%$, a positive predictive value of $75 \%$, and a negative predictive value of $98.6 \%{ }^{38}$

Other investigators, however, have found much higher false positive rates. When Pickering et al tested and compared serum and plasma samples from 36 healthy blood donors with 15 patients with candidemia, all the samples from the blood donors were negative for BDG and 13 of 15 candidemic patients were positive for BDG. However, 14 of 25 patients with bacteremia were also positive for BDG. They concluded that the test was likely more useful for excluding an IFI. ${ }^{39}$

There are few studies addressing specifically the role of BDG after liver transplantation. Chen et al planned and drew blood for procalcitonin and BDG in 55 patients with suspected catheter-related bloodstream infections after orthotopic liver transplantation. Unfortunately, they had only one case of fungemia; hence, conclusions about the role of BDG in liver transplant patients cannot be drawn from their study. ${ }^{40}$
Yamanouchi et al measured BDG levels regularly after living donor liver transplantation. Seventy-one patients had 89 episodes of elevated BDG; $47 \%$ of the cases of raised BDG occurred in the first 5 days post-transplant. ${ }^{41}$ This is not surprising as false positive BDG elevation has been associated with the infusion of albumin and globulins, with hemodialysis, and even with surgical gauze - all of which are not uncommon in liver transplant patients in the early postoperative period. Patients with elevated levels of BDG were more likely to have a positive fungal culture, but only one of these was a positive blood culture. The most significant finding in this study, however, was the higher mortality rate in patients with raised BDG levels after the 15 th postoperative day.

Although mannan/antimannan assays have also been recommended for the diagnosis of candidemia, hardly any study has used them in the field of liver transplant. In a landmark paper that showed that using immunoassays detecting both the mannan antigen and the antimannan antibody improved the sensitivity of the assay, Sendid et al included only one patient whose underlying condition was "liver transplantation". ${ }^{42}$ In another publication, the same group suggested that regular monitoring of mannanemia and antimannan antibodies in patients at risk of candidiasis might facilitate its early diagnosis. ${ }^{43}$

Antifungal susceptibility is generally predicted on the basis of species and local epidemiology. However, given the increasing use of antifungals and emerging resistant Candida spp., it is reasonable to request antifungal susceptibility testing of isolates when resistant fungi or treatment failure is suspected. ${ }^{23}$

\section{Prevention of Candida infections}

Liver transplant recipients are at a constant threat of infections depending on their net state of immunosuppression. Preventing infections is a multipronged approach and requires the collaborative effort of teams managing these immunocompromised hosts.

\section{Donor-derived Candida infections}

A wide range of infections have been transmitted from donors to transplant recipients and are increasingly being recognized to be associated with significant post-transplant morbidity and mortality. ${ }^{44,45}$ The Organ Procurement and Transplant Network in the US is the currently available database of the estimated donor-derived infectious disease transmissions among solid organ transplants. Thirty fungal infections including Candida spp. were reported between 
2005-2009 that were potentially donor derived. ${ }^{44}$ Most cases occurred in kidney and liver recipients with contaminated preservation fluid being implicated as the source. ${ }^{46}$ Preventive strategies include monitoring and culturing the organ preservation media and avoiding organs from patients with active infections. Potential donors, especially the cadaveric donors, are ill days to weeks before their organs are procured, and are cared for in the intensive care units, and are at risk for IFIs. The evaluation of potential deceased donors for infections includes blood and urine cultures, which the authors suggest for potential donors with problems like fever or leukocytosis.

\section{Immunization}

Vaccination is one of the effective strategies to prevent infections. In solid organ transplants, just like in other immunocompromised hosts, the risk of acquiring and also the ability to mount protective immune response depends on the net state of immunosuppression. ${ }^{3}$ The American Society of Transplantation has published guidelines which include vaccination of solid organ transplant candidates/recipients and also household members and health care workers who are potential sources of transmitting infections. ${ }^{47}$ Currently, there are no known approved vaccines against fungal infections. ${ }^{48}$ There are ongoing efforts to develop antifungal vaccines; two Phase I clinical trials against Candida spp. have been evaluated. ${ }^{49,50}$ There are concerns with regards to the preclinical studies of the antifungal research as the majority use mouse models, which have different innate immune response when compared to humans. ${ }^{48} \mathrm{New}$ developments looking at a conjugate vaccine that elicits antibodies to BDG against C. albicans, Aspergillus fumigatus, and Cryptococcus neoformans and inducing immunity by vaccinating with heat-killed Saccharomyces cerevisiae are two of the pan fungal vaccination options that are being pursued and may be anticipated in the future to help prevent IFIs. ${ }^{51,52}$

\section{Prophylaxis}

Current consensus and recommendation is for antifungal prophylaxis in liver transplant candidates at high risk for candidiasis. This is a practice well supported by the data. Cruciani et al found that prophylaxis reduced colonization, total proven fungal infections (including invasive infections), and mortality attributable to fungal infection. ${ }^{17} \mathrm{~A}$ Cochrane review found that fluconazole reduced the incidence of IFIs without a mortality benefit and suggested that individuals at greatest risk be selected for prophylaxis. ${ }^{53}$ As the likelihood of IC is greatest in the early post-transplant period, this is the period generally chosen for antifungal prophylaxis. ${ }^{12}$ There is less consensus, however, on the agent of choice, as shown in a survey of transplant units. ${ }^{7}$ Although certain units practice universal prophylaxis, it has been shown that this has not affected overall mortality; furthermore, there is a risk of developing infection with non-albicans Candida spp. ${ }^{16-18}$ Hence, perhaps in line with the Cochrane review, the practice of "targeted prophylaxis" seems to be most popular, with patients defined as being at risk selected for prophylaxis. Although "risk" is not easy to define, Pappas et al found that in patients without any, or only one, of the following risk factors prophylaxis could safely be withheld: choledochojejunostomy anastomosis, retransplantation, intraoperative administration of $\geq 40$ units of blood products or return to the operating room for intraabdominal bleeding, return to the operating room for anastomotic leak or vascular insufficiency, preoperative serum creatinine of $\geq 2 \mathrm{mg} / \mathrm{dL}$, and perioperative Candida colonization. ${ }^{16}$ In clinical practice, the presence of at least two of these risk factors would usually indicate prophylaxis.

Model of End-Stage Liver Disease (MELD) score was first developed in 2000 to predict mortality of patients undergoing transjugular intrahepatic portosystemic shunts and is now widely used to prioritize patients who require liver transplantation. Prognostic value of the MELD score has been assessed both for bacterial infections and post-transplant survival. In a recent paper from a single center, retrospective analysis of 667 liver transplants identified that MELD scores of 20-30 or $\geq 30$ were associated with a 2.0 -fold or 4.3 -fold increase in relative risk of fungal infections. ${ }^{54} \mathrm{It}$ is postulated from this observation that the routinely calculated MELD score may serve as a simple and practical tool to predict the risk for infections and at the same time identify candidates for antifungal prophylaxis.

The American Society of Transplantation and the American Society of Transplant Surgeons recommend fluconazole as the antifungal prophylaxis of choice against candidiasis followed by lipid formulations of amphotericin B in centers with high rates of non-albicans Candida spp. Caspofungin is being used for antifungal prophylaxis in liver transplant recipients. ${ }^{7}$ Echinocandins may become the antifungal prophylaxis of choice for liver transplant recipients, given the efficacy and safety demonstrated in high-risk liver transplant patients combined with the increasing incidence of infections due to non-albicans Candida spp. ${ }^{55}$

There is no consensus with regards to the duration of antifungal prophylaxis. Given the paucity of studies in defining the duration of antifungal prophylaxis, Pappas and 
Silveira recommend $\geq 4$ weeks or as long as the risk period warrants prophylaxis. ${ }^{55}$

\section{Antimicrobial stewardship programs}

The scope of antimicrobial stewardship programs in the management of infections is expanding. ${ }^{56,57}$ This is timely given the surveillance studies showing an increase in IC in both intensive care units and nonintensive care settings. With broad-spectrum antibiotic use a recognized risk factor for candidiasis, antimicrobial stewardship programs may help to prevent IFIs. More recently, antimicrobial stewardship programs are monitoring the use of antifungal drugs which may improve the management of IFIs and also prevent the emergence of resistant Candida spp. ${ }^{58,59}$

\section{Infection control}

Nosocomial fungal infections caused by Candida spp. and Aspergillus spp. are increasingly being recognised. ${ }^{60}$ The National Healthcare Safety Network data collected during 2009-2010 from US hospitals reports Candida spp. features among the top eight pathogens (Staphylococcus aureus, Enterococcus spp., Escherichia coli, coagulase-negative staphylococci, Klebsiella pneumonia, Klebsiella oxytoca, and Klebsiella pseudomonas, and Enterobacter spp.) that are major contributors to health care-associated infections. ${ }^{61}$ Candida spp. rank as the third most common cause of central line-associated bloodstream infections in US intensive care units. ${ }^{62}$ Among solid organ transplant recipients, candidemia compared to the Gram-positive and Gram-negative bloodstream infections was associated with a higher crude mortality. ${ }^{4}$ Nosocomial transmission of Candida spp. from health care workers to patients has been documented and also implicated in outbreaks. ${ }^{60}$ Infection control programs with the goal of reducing health care-associated infections and thereby improving patient safety have grown since the results of the Study on the Efficacy of Nosocomial Infections and Infection Control Practices (SCENIC). ${ }^{63}$ Strategies to prevent central line-associated bloodstream infections, which include educating health care personnel about central line care, ensuring adherence to infection prevention practices at time of insertion (attention to hand hygiene, avoiding femoral veins, maximal sterile barrier precautions, chlorhexidinebased antiseptic for skin preparation), disinfecting catheter hubs prior to each use, and removing nonessential catheters, are some of the current recommendations from the Society of Healthcare Epidemiology of America and Infectious Diseases Society of America. ${ }^{64}$ There is currently no evidence to support the isolation of patients with Candida colonization or infection. ${ }^{65}$ Hand washing is recognized as an important and effective procedure for preventing health care-associated infections, recognizing the fact that Candida spp. are carried on the hands of health care workers. ${ }^{66}$

\section{Treatment of Candida infections}

Empiric treatment with an anti-Candida agent for suspected IC followed by definitive therapy by selecting the appropriate agent based on the species of Candida isolated and susceptibility profile, if available, is recommended. ${ }^{27} \mathrm{~A}$ delay in starting antifungal treatment has been associated with increased mortality. ${ }^{67}$ Four categories of antifungal agents are available and all are effective for treating Candida infections: the polyenes (amphotericin B, deoxycholate amphotericin, liposomal amphotericin B, amphotericin B lipid complex, and amphotericin B colloidal dispersion), the triazoles (fluconazole, itraconazole, voriconazole, and posaconazole), the echinocandins (caspofungin, anidulafungin, and micafungin), and flucytosine. The treatment guidelines from the Infectious Diseases Society of America for managing candidiasis are also recommended for liver transplant recipients. ${ }^{15,68}$

Improved survival associated with echinocandin use from reviewing seven randomized antifungal treatment trials for IC has led to a more definite consensus and reiterates the Infectious Diseases Society of America recommendations of using echinocandins for most patients with IC. ${ }^{69}$ Caspofungin, micafungin, and anidulafungin are the three licensed echinocandins which have turned out to be valuable options in the treatment of fungal infections in the 21 st century. ${ }^{70}$ Their popularity seems to be well justified: they have broad candidacidal activity compared to the triazoles (they are effective against fluconazole-resistant Candida spp.), they have an excellent side effect profile (with low potential for nephrotoxicity and hepatotoxicity), and they did very well in the early trials (achieving superiority over comparators in some of the studies)..$^{71,72}$

With the availability of more antifungal drugs, several important points should be borne in mind when treating patients with candidemia/IC. Emergence of resistant fungi and breakthrough IFIs in patients already on antifungals has been reported in liver transplant recipients. These include resistant $C$. glabrata strains while on azoles, breakthrough C. glabrata and C. parapsilosis while on micafungin, and trichosporonosis while on caspofungin. ${ }^{73-75}$ Pharmacokinetics and pharmacodynamics of antifungal drugs vary and should be considered while treating certain IFIs, especially those involving the heart, central nervous system, and eyes; the use of intravenous or oral formulations depending on the 
patients clinical status and drug-drug interactions while on immunosuppressants and concomitant medications should also be considered.

\section{Conclusion}

Candida remains the most common fungal infection in liver transplant recipients. Antifungal prophylaxis with fluconazole or an echinocandin for selected patients at higher risk for candidemia will likely help to reduce the risk of IC. Diagnosis is difficult. Blood cultures, although of modest sensitivity, are a must. The role of nonculture diagnostics such as BDG needs to be formally studied in liver transplant recipients. Early treatment with an echinocandin is recommended, although the risk of breakthrough infections should be recognized.

\section{Disclosure}

The authors report no conflicts of interest in this work.

\section{References}

1. Paya CV, Hermans PE, Washington JA 2nd, et al. Incidence, distribution, and outcome of episodes of infection in 100 orthotopic liver transplantations. Mayo Clin Proc. 1989;64(5):555-564.

2. Arnow PM. Infections following orthotopic liver transplantation. $H P B$ Surgery. 1991;3(4):221-233.

3. Fishman JA. Infections in solid-organ transplant recipients. $N$ Engl J Med. 2007;357(25):2601-2614.

4. Moreno A, Cervera C, Gavalda J, et al. Bloodstream infections among transplant recipients: results of a nationwide surveillance in Spain. Am J Transplant. 2007;7(11):2579-2586.

5. Watt KD, Pedersen RA, Kremers WK, Heimbach JK, Charlton MR. Evolution of causes and risk factors for mortality post-liver transplant: results of the NIDDK long-term follow-up study. Am J Transplant. 2010;10(6):1420-1427.

6. Rubin RH. Overview: pathogenesis of fungal infections in the organ transplant recipient. Transpl Infect Dis. 2002;4(Suppl 3):12-17.

7. Singh N, Wagener MM, Cacciarelli TV, Levitsky J. Antifungal management practices in liver transplant recipients. Am J Transplant. 2008;8(2):426-431.

8. Eschenauer GA, Lam SW, Carver PL. Antifungal prophylaxis in liver transplant recipients. Liver Transpl. 2009;15(8):842-858.

9. Singh N, Wagener MM, Marino IR, Gayowski T. Trends in invasive fungal infections in liver transplant recipients: correlation with evolution in transplantation practices. Transplantation. 2002;73(1):63-67.

10. Romero FA, Razonable RR. Infections in liver transplant recipients. World J Hepatol. 2011;3(4):83-92.

11. Fung JJ. Fungal infections in liver transplantation. Transpl Infect Dis. 2002;4(Suppl 3):18-23.

12. Pappas $P G, A$ Alexander $B D$, Andes DR, et al. Invasive fungal infections among organ transplant recipients: results of the Transplant-Associated Infection Surveillance Network (TRANSNET). Clin Infect Dis. 2010;50(8):1101-1111.

13. Husain S, Tollemar J, Dominguez EA, et al. Changes in the spectrum and risk factors for invasive candidiasis in liver transplant recipients: prospective, multicenter, case-controlled study. Transplantation. 2003;75(12):2023-2029.

14. Neofytos D, Fishman JA, Horn D, et al. Epidemiology and outcome of invasive fungal infections in solid organ transplant recipients. Transpl Infect Dis. 2010;12(3):220-229.
15. Shoham S, Marr KA. Invasive fungal infections in solid organ transplant recipients. Future Microbiol. 2012;7(5):639-655.

16. Pappas PG, Andes D, Schuster M, et al. Invasive fungal infections in low-risk liver transplant recipients: a multi-center prospective observational study. Am J Transplant. 2006;6(2):386-391.

17. Cruciani M, Mengoli C, Malena M, Bosco O, Serpelloni G, Grossi P. Antifungal prophylaxis in liver transplant patients: a systematic review and meta-analysis. Liver Transpl. 2006;12(5):850-858.

18. Pfaller MA, Messer SA, Moet GJ, Jones RN, Castanheira M. Candida blood stream infections: comparison of species distribution and resistance to echinocandin and azole antifungal agents in intensive care unit (ICU) and non-ICU settings in the SENTRY Antimicrobial Surveillance Program (2008-2009). Int J Antimicrob Agents. 2011;38(1):65-69.

19. Kett DH, Azoulay E, Echeverria PM, Vincent JL. Candida blood stream infections in intensive care units: analysis of the extended prevalence of infection in intensive care unit study. Crit Care Med. 2011;39(4): 665-670.

20. Yang YL. Virulence factors of Candida species. J Microbiol Immunol Infect. 2003;36(4):223-228.

21. Silva S, Negri M, Henriques M, Oliveira R, Williams DW, Azeredo J. Adherence and biofilm formation of non-Candida albicans Candida species. Trends Microbiol. 2011;19(5):241-247.

22. Ganguly S, Mitchell AP. Mucosal biofilms of Candida albicans. Curr Opin Microbiol. 2011;14(4):380-385.

23. Lockhart SR, Wagner D, Iqbal N, et al. Comparison of in vitro susceptibility characteristics of Candida species from cases of invasive candidiasis in solid organ and stem cell transplant recipients: TransplantAssociated Infections Surveillance Network (TRANSNET), 2001 to 2006. J Clin Microbiol. 2011;49(7):2404-2410.

24. Fishman JA, Rubin RH. Infections in organ-transplant recipients. N Engl J Med. 1998;338(24):1741-1751.

25. Low CY, Rotstein C. Emerging fungal infections in immunocompromised patients. F1000 Med Rep. 2011;3:14.

26. Rubin RH. Fungal infections in the organ transplant recipient. In: Anaissie EJ, McGinnis MR, Pfaller MA, editors. Clinical Mycology. 2nd ed. London: Churchill Livingstone; 2009:471-478.

27. Pappas PG, Kauffman CA, Andes D, et al. Clinical practice guidelines for the management of candidiasis: 2009 update by the Infectious Diseases Society of America. Clin Infect Dis. 2009;48(5): 503-535.

28. Safdar N, Said A, Lucey MR, et al. Infected bilomas in liver transplant recipients: clinical features, optimal management, and risk factors for mortality. Clin Infect Dis. 2004;39(4):517-525.

29. Asensio A, Ramos A, Cuervas-Mons V, et al. Effect of antibiotic prophylaxis on the risk of surgical site infection in orthotopic liver transplant. Liver Transplantation. 2008;14(6):799-805.

30. Reid GE, Grim SA, Sankary H, Benedetti E, Oberholzer J, Clark NM. Early intra-abdominal infections associated with orthotopic liver transplantation. Transplantation. 2009;87(11):1706-1711.

31. Hellinger WC, Crook JE, Heckman MG, et al. Surgical site infection after liver transplantation: risk factors and association with graft loss or death. Transplantation. 2009;87(9):1387-1393.

32. Singh N. The current management of infectious diseases in the liver transplant recipient. Clin Liver Dis. 2000;4(3):657-673.

33. de Pauw B, Walsh TJ, Donnelly JP, et al. Revised definitions of invasive fungal disease from the European Organization for Research and Treatment of Cancer/Invasive Fungal Infections Cooperative Group and the National Institute of Allergy and Infectious Diseases Mycoses Study Group (EORTC/MSG) Consensus Group. Clin Infect Dis. 2008;46(12): 1813-1821.

34. Ascioglu S, Rex JH, de Pauw B, et al. Defining opportunistic invasive fungal infections in immunocompromised patients with cancer and hematopoietic stem cell transplants: an international consensus. Clin Infect Dis. 2002;34(1):7-14.

35. Cuenca-Estrella M, Verweij PE, Arendrup MC, et al. ESCMID guideline for the diagnosis and management of Candida diseases 2012: diagnostic procedures. Clin Microbiol Infect. 2012;18(Supp1 7):9-18. 
36. Hall L, Le Febre KM, Deml SM, Wohlfiel SL, Wengenack NL. Evaluation of the yeast traffic light PNA FISH probes for identification of candida species from positive blood cultures. J Clin Microbiol. 2012;50(4):1446-1448.

37. Spanu T, Posteraro B, Fiori B, et al. Direct maldi-tof mass spectrometry assay of blood culture broths for rapid identification of Candida species causing bloodstream infections: an observational study in two large microbiology laboratories. J Clin Microbiol. 2012;50(1):176-179.

38. Posteraro B, De Pascale G, Tumbarello M, et al. Early diagnosis of candidemia in intensive care unit patients with sepsis: a prospective comparison of $(1 \rightarrow 3)-\beta-D$-glucan assay, Candida score, and colonization index. Crit Care. 2011;15(5):R249.

39. Pickering JW, Sant HW, Bowles CA, Roberts WL, Woods GL. Evaluation of a $(1 \rightarrow 3)-\beta$-D-glucan assay for diagnosis of invasive fungal infections. J Clin Microbiol. 2005;43(12):5957-5962.

40. Chen J, Wang Y, Shen Z, Zhu Z, Song Y, Han R. Early diagnostic value of plasma PCT and BG assay for CRBSI after OLT. Transplant Proc. 2011;43(5):1777-1779.

41. Yamanouchi K, Takatsuki M, Hidaka M, Soyama A, Kanematsu T, Eguchi S. Significance of serum $\beta$-D-glucan levels in recipients of living donor liver transplantation. J Hepatobiliary Pancreat Sci. 2011;18(3): 432-435.

42. Sendid B, Tabouret M, Poirot JL, Mathieu D, Fruit J, Poulain D. New enzyme immunoassays for sensitive detection of circulating Candida albicans mannan and antimannan antibodies: useful combined test for diagnosis of systemic candidiasis. J Clin Microbiol. 1999;37(5): $1510-1517$.

43. Sendid B, Poirot JL, Tabouret M, et al. Combined detection of mannanemia and antimannan antibodies as a strategy for the diagnosis of systemic infection caused by pathogenic Candida species. $J$ Med Microbiol. 2002;51(5):433-442.

44. Ison MG, Nalesnik MA. An update on donor-derived disease transmission in organ transplantation. Am J Transplant. 2011;11(6):1123-1130.

45. Greenwald MA, Kuehnert MJ, Fishman JA. Infectious disease transmission during organ and tissue transplantation. Emerg Infect Dis. 2012;18(8):e1

46. Singh N, Huprikar S, Burdette SD, Morris MI, Blair JE, Wheat LJ. Donor-derived fungal infections in organ transplant recipients: guidelines of the American Society of Transplantation, Infectious Diseases Community of Practice. Am J Transplant. 2012;12(9):2414-2428.

47. Danzinger-Isakov L, Kumar D. Vaccination in solid organ transplantation. Am J Transplant. 2013;13(Suppl 4):311-317.

48. Edwards JE Jr. Fungal cell wall vaccines: an update. J Med Microbiol. 2012;61(Pt 7):895-903.

49. Schmidt CS, White CJ, Ibrahim AS, et al. NDV-3, a recombinant alumadjuvanated vaccine for Candida and Staphylococcus aureus, is safe and immunogenic in healthy adults. Vaccine. 2012;30(52):7594-7600.

50. Sandini S, La Valle R, Deaglio S, Malavasi F, Cassone A, De Bernardis F. A highly immunogenic recombinant and truncated protein of the secreted aspartic proteases family (rSap2t) of Candida albicans as a mucosal anticandidal vaccine. FEMS Immunol Med Microbiol. 2011;62(2):215-224.

51. Torosantucci A, Chiani P, Bromuro C, et al. Protection by anti- $\beta$ glucan antibodies is associated with restricted $\beta-1,3$ glucan binding specificity and inhibition of fungal growth and adherence. PLoS One. 2009;4(4):e5392.

52. Stevens DA, Clemons KV, Liu M. Developing a vaccine against Aspergillosis. Med Mycol. 2011;49(Suppl 1):170-176.

53. Playford EG, Webster AC, Sorell TC, Craig JC. Antifungal agents for preventing fungal infections in solid organ transplant recipients [review]. Cochrane Database Syst Rev. 2004;3:CD004291.

54. Saliba F, Delvart V, Ichai P, et al. Fungal infections after liver transplantation: outcomes and risk factors revisited in the MELD era. Clin Transplant. 2013;27(4):E454-E461.

55. Pappas PG, Silveira FP. Candida in solid organ transplant recipients. Am J Transplant. 2009;9(Suppl 4):S173-S179.
56. Goff DA, Bauer KA, Mangino JE. Antimicrobial Stewardship Management of Infections: Beyond the Costs of Antimicrobials. New York, NY: Pharmacy Practice News; 2012. Available from: http://www.pharmacypracticenews.com/download/Antimicrobial_ stewardship_WM.pdf. Accessed June 5, 2014.

57. Reed EE, West JE, Keating EA, et al. Improving the management of candidemia through antimicrobial stewardship interventions. Diagn Microbiol Infect Dis. 2014;78(2):157-161.

58. Amanda-Rajah MR, Slavin MA, Thursky KT. The case for antifungal stewardship. Curr Opin Infect Dis. 2012;25(1):107-115.

59. Ruhnke M. Antifungal stewardship in invasive Candida infections. Clin Microbiol Infect. 2014;20(Suppl 6):11-18.

60. Alangaden GJ. Nosocomial fungal infections: epidemiology, infection control, and prevention. Infect Dis Clin North Am. 2011;25(1): 201-225.

61. Sievert DM, Ricks P, Edwards JR, et al. Antimicrobial-resistant pathogens associated with healthcare-associated infections: summary of data reported to the National Healthcare Safety Network at the Centers of Disease Control and Prevention, 2009-2010. Infect Control Hosp Epidemiol. 2013;34(1):1-14.

62. Hidron AI, Edwards JR, Patel J, et al. Antimicrobial-resistant pathogens associated with healthcare-associated infections: annual summary of data reported to National Healthcare Safety Network at the Centers for Disease Control and Prevention, 2006-2007. Infect Control Hosp Epidemiol. 2008;29(11):996-1011.

63. Sydnor ER, Perl TM. Hospital epidemiology and infection control in acute-care settings. Clin Microbiol Rev. 2011;24(1):141-173.

64. Marschall J, Mermel LA, Classen D, et al. Strategies to prevent central line-associated bloodstream infections in acute care hospitals. Infect Control Hosp Epidemiol. 2008;29(Suppl 1):S22-S30.

65. Pammi M, Eddama O, Weisman LE. Patient isolation measures for infants with Candida colonization or infection for preventing or reducing transmission of Candida in neonatal units [review]. Cochrane Database Syst Rev. 2011;11:CD006068

66. Tomblyn M, Chiller T, Einsele H, et al. Guidelines for preventing infectious complications among hematopoietic cell transplantation recipients: a global perspective. Biol Blood Marrow Transplant. 2009;15(10): 1143-1238.

67. Morrell M, Fraser VJ, Kollef MH. Delaying the empiric treatment of Candida bloodstream infection until positive blood culture results are obtained: a potential risk factor for hospital mortality. Antimicrob Agents Chemother. 2005;49(9):3640-3645.

68. Silveira FP, Kusne S. Candida infections in solid organ transplantation. Am J Transplant. 2013;13(Suppl 4):220-227.

69. Andes DR, Safdar N, Baddley JW, et al. Impact of treatment strategy on outcomes in patients with candidemia and other forms of invasive candidiasis: a patient-level quantitative review of randomized trials. Clin Infect Dis. 2012;54(8):1110-1122.

70. Chen SC, Slavin MA, Sorrell TC. Echinocandin antifungal drugs in fungal infections: a comparison. Drugs. 2011;71(1):11-41.

71. Mora-Duarte J, Betts R, Rotstein C, et al. Comparison of caspofungin and amphotericin B for invasive candidiasis. $N$ Engl $J$ Med. 2002;347(25):2020-2029.

72. Reboli AC, Rotstein C, Pappas PG, et al. Anidulafungin versus fluconazole for invasive candidiasis. N Engl J Med. 2007;356(24):2472-2482.

73. Fortun J, Lopez-San Roman A, Velasco JJ, et al. Selection of Candida glabrata strains with reduced susceptibility to azoles in four liver transplant patients with invasive candidiasis. Eur J Clin Microbiol Infect Dis. 1997;16(4):314-318.

74. Pfeiffer CD, Garcia-Effron G, Zaas AK, Perfect JR, Perlin DS, Alexander BD. Breakthrough invasive candidiasis in patients on micafungin. J Clin Microbiol. 2010;48(7):2373-2380.

75. Lingegowda PB, Tan CK, Tan AL, Tan BH. Selection of resistant fungi in liver transplant recipients during use of newer antifungal agents: a report of two cases. Ann Acad Med Singapore. 2011;40(6): $287-290$. 
Transplant Research and Risk Management

Dovepress

\section{Publish your work in this journal}

Transplant Research and Risk Management is an international, peerreviewed open access journal focusing on all aspects of transplantation and risk management to achieve optimal outcomes in the recipient improving survival and quality of life. The journal welcomes submitted papers covering original research, basic science, clinical studies,

reviews \& evaluations, guidelines, expert opinion and commentary, case reports and extended reports. The manuscript management system is completely online and includes a very quick and fair peer-review system, which is all easy to use. Visit http://www.dovepress.com/ testimonials.php to read real quotes from published authors.

Submit your manuscript here: http://www.dovepress.com/transplant-research-and-risk-management-journal 